\title{
Identità oltre l'illusione Palestina, terra di architettura e poteri, dove smarrirsi può significare trovare senso e identità
}

\author{
di Virginia Fiume
}

MA Antropologia dei Media - School of Oriental and African Studies virginia.fiume@gmail.com

www.virginiafiume.com

\begin{abstract}
Il caso di studio presentato è relativo al contesto israelo-palestinese e cerca di dimostrare con esempi pratici e teorici come architettura e urbanistica siano elementi che mettono in pratica una relazione di potere e che l'approccio situazionista costituisce lo strumento principe per superare questa relazione. Solo dopo questo momento di superamento si può costruire una comunicazione alternativa, forte al punto da diventare contro-egemonica. Nella prima parte dell'articolo vengono forniti gli strumenti teorici sulle interpretazioni del concetto di paesaggio dal punto di vista antropologico, sociologico e geografico. La seconda parte inserisce queste teorie all'interno di esemplificazioni pratiche sull'urbanistica e la simbologia burocratica in Israele e Palestina, con particolare riferimento al concetto di "matrix of control" elaborato dall'antropologo e attivista israeloamericano Jeff Halper. Il cuore dell'articolo sarà la presentazione di una parte di una ricerca etnografica svolta dall'autrice nell'estate del 2011 nei Territori Palestinesi Occupati. In particolare si racconta di un viaggio in macchina svolto tra Betlemme e Jenin (al limite nord della Cisgiordania).

The case study is focused on the Israeli-Palestinian context. Power relations are analyzed under the lenses of practical examples and bibliographic references to demonstrate how in this peculiar context architecture and urban planning constitute a web of power. A situationist approach has been suggested as a key tool to overcome those power relations. It is just in virtue of this situationist approach that an alternative discourse can grow, becoming strong enough to be a counter-hegemonic narrative. The first part of the essay set up a set of interpretive tools, analysing the concept of landscape from an anthropological, sociological and geographical perspective. The second part merge those theories with practical samples taken from Israeli and Palestinian bureaucracy and urban planning. A key concept is the "matrix of control" proposed by the Israeli-American anthropologist and activist Jeff Halper. The core of the essay is an excerpt of the fieldwork diary of the author, written during summer 2011 in the Occupied Palestinian Territories. The detail of the account is a car trip from Bethlehem to Jenin (on the North shore of West Bank).
\end{abstract}

\section{Parole chiave}

Turismo, urbanistica, memoria, paesaggio, situazionismo

Tourism, urban planning, memory, landscape, situationism

\section{Sommario}

o. Introduzione

1. Il paesaggio come luogo delle interpretazioni

2. Paesaggio, ambiente e controllo

3. L'industria dell'illusione

4. Smarrirsi per sfuggire al controllo

Bibliografia 


\begin{abstract}
Ma come era venuta, a Erodoto, quella passione? Forse tutto era cominciato da una di quelle domande che fanno i bambini: "Da dove arrivano le navi?”. Giocando con la sabbia in riva al mare, i bambini vedono improvvisamente spuntare all'orizzonte una nave che, venendo verso di loro, diventa sempre più grande. Ma da dove viene? Sono domande che di solito la maggior parte di essi non si pone. Di tanto in tanto però ce n'è uno che, costruendo il suo castello di sabbia, chiede: "Ma da dove arriva quella nave? Quella linea lontana sembrava la fine del mondo: possibile che dietro di essa ci sia un altro mondo e, dietro, un altro ancora? e di che mondi si tratta?”. Il bambino comincia a cercare una risposta e, una volta diventato grande, la cerca con sempre più insistenza e curiosità.
\end{abstract}

Ryszard Kapuscinski, In viaggio con Erodoto

\section{o. Introduzione}

"Una terra senza popolo per un popolo senza terra". È probabilmente la frase più famosa e il più famoso luogo comune della narrazione dominante sul cosiddetto conflitto israelo-palestinese. Nell'immaginario collettivo questa frase ha giustificato la decisione della comunità internazionale di insediare il progetto sionista nell'area della Palestina storica, fino al 1947 sotto il controllo del Mandato Britannico. Come tutti i luoghi comuni, la frase, dal vago sapore coloniale, è un punto di partenza interessante per una analisi antropologica e geografica sul paesaggio.

Il cuore di questo saggio sarà il paesaggio in Terra Santa, che diventa caso di studio per tentare di comprendere come il concetto di "contested landscape"1 (paesaggio conteso) elaborato da Barbara Bender possa essere un concetto chiave per approcciarsi a Israele e ai Territori Palestinesi Occupati. La questione acquista rilevanza globale se si considera che l'area è una delle più famose mete di pellegrinaggio e viaggio dell'intero bacino mediterraneo.

Lo studio è stato diviso in quattro sezioni. La prima parte prende in esame il concetto di "contested landscape", cercando di fare alcuni cenni al più ampio contesto dell'antropologia del paesaggio, così come teorizzata nel 1995 da Eric Hirsch in The Anthropology of landscape. Perspective on place and space. La seconda parte descrive il sistema di relazioni di potere in cui si articola il paesaggio palestinese intersecandosi con quello israeliano. I testi base sono stati la recente ricerca Surveillance and control in Israel/Palestine e il lavoro dell'antropologo e attivista israeliano Jeff Halper. La terza parte definisce le caratteristiche dell'industria del turismo locale, che la fanno definire "industria dell'illusione", analizzando casi concreti di manipolazione del paesaggio attraverso strumenti come le guide e gli investimenti economici. La quarta parte dimostra come lo smarrimento

$1 \quad$ Il termine viene usato qui e in tutto il testo in inglese perché la fonte primaria dell'articolo è l'introduzione di Barbara Bender al volume intitolato Contested Lanscape. Movement, exile and place, 2001 
generato da questi tre fattori può diventare una via di fuga, una tecnica di contro-potere. Alle teorie situazioniste di Guy Debord viene affiancato un aneddoto, frutto di parte della ricerca sul campo da me effettuata nell'estate del 2011 per l'elaborazione della tesi From Holy Land to Palestine. The role of "international committed people" in the Palestinian struggle.

Quello che cercherò di dimostrare con questo lavoro di ricerca e con la presentazione di un frammento di osservazione partecipativa è che il paesaggio di Israele e dei Territori Palestinesi Occupati è espressione di una relazione di potere tra una forza oppressiva (il Governo dello Stato di Israele) e una popolazione oppressa (il popolo palestinese e, per certi aspetti, anche la popolazione israeliana e i viaggiatori). Ma guarderò con ottimismo a questa rete, descrivendo il modo in cui il corpo e il movimento casuale, a volte frutto proprio della ragnatela urbanistico architettonica, giochino un ruolo decisivo e vincente in questa relazione.

\section{Il paesaggio come luogo delle interpretazioni}

Nell'introduzione di The Anthropology of Landscape. Perspective on place and space Eric Hirsch invita chi studia il paesaggio da un punto di vista antropologico a identificare due campi di analisi ben precisi. Se da una parte Hirsch definisce il paesaggio come una "framing convention" (una convenzione racchiusa in una cornice), per altri versi lo considera un insieme complesso di significati proiettati al suo interno dalle popolazioni locali, secondo gli elementi culturali e fisici in cui queste sono immerse. All'interno di questa seconda interpretazione Hirsch aggiunge due ulteriori livelli di analisi: il paesaggio può essere a sua volta suddiviso in "primo piano", luogo deputato al "contingente", e in "sfondo", casa del "potenziale". È Hirsch stesso a specificare che queste due componenti coesistono, come momenti transitori.

Ritengo questo gioco di alternanze di differenti prospettive uno degli elementi determinanti dell'uso del paesaggio che è stato fatto sull'area oggetto di questa analisi nel corso della storia, fino ai giorni nostri. Il migliore esempio viene proprio dal luogo comune utilizzato all'inizio di questo racconto: la Palestina storica è stata descritta da viaggiatori e storiografi come una terra deserta, dove all'inizio del ventesimo secolo i coloni ebrei sono stati legittimati e incoraggiati a recarsi per realizzare il loro progetto: "porta il progresso", "far fiorire il deserto". ${ }^{2}$

Questa narrazione, di cui si trovano esempi in autori come Mark Twain, citati come fonti dalla storiografia sionista, sceglie di ignorare la

2 Per comprendere quanto queste frasi siano persistenti nel discorso comune contemporaneo si può prendere come riferimento l'intervista che Claudio Pagliara, corrispondente del TG1 del servizio pubblico italiano a Shimon Peres andata in onda in un'edizione del telegiornale del maggio 2011 e trascritta e pubblicata dal giornalista stesso sul suo blog. Si legge: "I problemi del Medio Oriente sono la povertà, la mancanza di acqua. Non c’è soluzione senza tecnologie avanzate. Guardi Israele: non ha acqua e non ha molta terra eppure ha una delle agricolture più avanzate del mondo". <www.claudiopagliara.it/2011/o5/intervista-a-shimon-peres-tg1-e-rai-news24> [ultimo accesso effettuato 10 giugno 2014] 
composizione demografica dell'area fino al 1917, anno della Dichiarazione di Balfour. ${ }^{3}$ L'area era abitata da popolazioni arabe che vivevano in maniera piuttosto armoniosa con popolazioni ebraiche. Si tratta della stessa narrazione che tende a non raccontare le azioni militari portate avanti dalle forze ebraiche (israeliane ante-litteram) che hanno contribuito in maniera significativa all'abbandono dell'area da parte dell'esercito britannico e all'intervento delle Nazioni Unite nell'elaborazione del cosiddetto Piano di Partizione 4 con cui fu sancita dalle Nazioni Unite la nascita di uno stato nazionale ebraico.

Un esempio recente di questo meccanismo che crea spazi vuoti in maniera tale da eliminare la versione alternativa del racconto si trova nell'opera di Rebecca Stein.5 Nel suo libro del 2008 la Stein descrive il periodo, a cavallo tra la fine degli anni Novanta, caratterizzati dallo scemare dell'ottimismo derivato dagli Accordi di Oslo, e l'inizio della Seconda Intifada, nel 2002. La sua analisi si concentra sulla copertura mediatica. A causa dei numerosi attacchi suicidi perpetrati dai combattenti palestinesi all'interno dei confini dello stato israeliano lo spazio urbano delle città ebraiche si era profondamente trasformato. Le persone preferivano, tendenzialmente, intrattenersi e rilassarsi in spazi privati, piuttosto che in luoghi pubblici. Uno degli attacchi più violenti colpì un elegante caffè a Gerusalemme Ovest, il Momentum. Secondo l'analisi della Stein, fu questo il momento in cui i media israeliani iniziarono a usare il paesaggio urbano israeliano, in particolare la potente immagine dei "caffè deserti", per farlo diventare una sorta di campo di battaglia, con i camerieri costretti a giocare il ruolo di agenti di sicurezza. Sebbene la Stein stessa non neghi che i caffè fossero effettivamente vuoti, ci offre anche un altro interessante punto di osservazione, dando a questo tropo un valore chiave nella narrazione sionista:

In parte il tropo è una sostituzione. Questa assenza era importantissima. Gli arabi palestinesi, che fossero residenti nei Territori Palestinesi Occupati o cittadini israeliani, erano assenti dalle città ebraiche. Pochissime persone usavano i mezzi di trasporto o entravano nei quartieri ebraici o nelle zone commerciali di Gerusalemme Ovest. Ai palestinesi residenti in Cisgiordania o a Gaza era proibito entrare in Israele dai checkpoint e da esigenze legate al visto. Un sentimento di rabbia anti araba stava montando, prendendo forma in slogan razzisti posti fuori dai negozi delle zone centrali, frasi come: "noi non assumiamo arabi" o "ai nemici non deve essere dato di che vivere”. Si sorvolava tranquillamente, nei media, sull'assenza dei palestinesi, usando l'assenza ebraica per oscurarli ulteriormente.

3 “Dichiarazione di Balfour” è il termine con cui viene identificato l'accordo del 1917 tra gli inglesi, potenza coloniale che controllava l'area della Palestina storica e l'organizzazione sionista.

4 Le fonti storiografiche cui si fa riferimento in tutto il saggio sono Ilan Pappe, $\mathrm{La}$ pulizia etnica della Palestina, Fazi, 2008 e James Gelvin, Il conflitto israelopalestinese. Cento anni di guerra, Einaudi, 2007.

5 La citazione si trova nel capitolo intitolato "Of cafés and terror" nel libro Itineraries of conflic. Israelis, Palestinians and the political lives of tourism, USA, Duke University Press. 
Se si considerano questi elementi e li si mette in relazione con la nozione di "contested landscape" così come è stata elaborata da Barbara Bender nel 2001 si può comprendere in che senso Israele, Gerusalemme e i Territori Palestinesi vengono a costituire un paesaggio di questo tipo. Per prima cosa perché, come afferma la Bender, i paesaggi non sono solo "contingenti dal punto di vista spaziale e storico", ma sono in continua trasformazione. Possiamo utilizzare un'altra categorizzazione, racchiusa in un altro saggio parte della raccolta curata da Barbara Bender: "intersecting landscape" (paesaggio incrociato), utilizzato da Thomas. Questa seconda definizione si riferisce a un contesto dove "due o più mondi diversi occupano uno stesso spazio fisico". Secondo Thomas la creazione di diversi paesaggi, tipicamente appartenenti alla stessa area geografica, è uno degli elementi che ci porta a considerare il paesaggio stesso come parte di un processo di soggettivazione.

Il risultato di questo processo in relazione al caso di studio oggetto di questa analisi è ben descritto dal politologo Meron Benvenisti. Lui stesso scrive, nella sua autobiografia ${ }^{6}$ di figlio di un geografo chiamato a mappare Eretz Israel (la terra di Israele), come l'incremento - simbolico e fisico - di possedimenti da parte degli ebrei israeliani fosse consequente a un esproprio ai danni dei palestinesi. Questa combinazione di appropriazioni ed espropriazioni ha portato a una situazione dove non esistono più tre dimensioni, ma sei: tre per i palestinesi e tre per gli israeliani.

A questi diversi paesaggi si aggiunge un ulteriore livello, quello dei paesaggi così come vengono percepiti e vissuti dai viaggiatori. I viaggiatori $\mathrm{e}$ le viaggiatrici vedono almeno tre dimensioni: il "paesaggio immaginario", il paesaggio della loro guida di riferimento e il paesaggio in cui si muovono con i loro corpi. Proprio quest'esperienza corporea è diversa per ogni viaggiatore, a seconda del tipo di itinerario che compie. Chi viaggia per godere della vita notturna di Tel Aviv probabilmente non assaporerà i luoghi sacri. Chi vive il territorio come Terra Santa probabilmente non incontrerà nessun palestinese o non vedrà i segni dell'occupazione. E a causa delle politiche di chiusura, turisti e viaggiatori non sono autorizzati ad entrare nella Striscia di Gaza. Nel terzo paragrafo mi riferirò a questa intelaiatura come "industria dell'illusione". Nel paragrafo seguente, invece, si descrive la rete di rapporti di potere.

\section{Paesaggio, ambiente e controllo}

Dopo il 1993, anno della firma degli Accordi di Oslo, il territorio dei Territori Palestinesi è stato diviso in diversi tipi di aree. Alcune sono state assegnate al completo controllo dell'Autorità Nazionale Palestinese (PA), identificate come "area A". Altre sono amministrate dalla PA e controllate militarmente dall'esercito israeliano. Altre ancora sono completamente gestite dalle forze militari o occupate dagli insediamenti civili israeliani.

6 L'autobiografia cui si fa riferimento si intitola Sacred landscape. The buried history of the Holy Land since 1948, University of California Press, Berkley. 
Questa suddivisione doveva essere temporanea, una fase della road map che avrebbe progressivamente portato al ritiro delle forze militari e civili israeliane dalla Cisgiordania e da Gaza, alla fine dell'occupazione militare e al progressivo congelamento delle colonie. Non è certo cosa nota a chi non segue quotidianamente il contesto israelo-palestinese il fatto che non solo questa road map non si è mai concretizzata, ma che la maggiore espansione delle colonie è avvenuta esattamente nei cinque anni immediatamente successivi agli accordi di Oslo.7 Adesso la popolazione ebraica all'interno delle aree palestinesi ha superato la cifra di 500.000 coloni.

A questo si aggiunge la recente mastodontica costruzione, la cosiddetta barriera di separazione. Il muro che secondo l'ideatore, Ariel Sharon, primo ministro di Israele tra il 2001 e il 2006, avrebbe dovuto separare Israele dai Territori Palestinesi e ridurre il numero di attentati terroristici in territorio israeliano. Il muro erode progressivamente le terre di proprietà dei palestinesi, limita la circolazione e l'accesso alle terre coltivate e coltivabili e causa un incremento considerevole delle demolizioni delle case.

Questo complesso di costruzioni, aree militari, zone cuscinetto (buffer zone) e strade preferenziali che collegano le colonie israeliane restando inaccessibili ai palestinesi ha spezzettato la Cisgiordania e Gerusalemme e la Striscia di Gaza a tal punto che il cartografo de L'atlas de le monde diplomatique ha definito la mappa "l'arcipelago".

Colonie, muro e checkpoint rappresentano l'evidenza fisica di questo colonialismo ibrido e della conseguente relazione di potere. Le colonie sono costruite sulla cima delle colline, secondo un progetto architettonico descritto da Eyal Weizman ${ }^{8}$ in gran parte del suo lavoro. Weizman applica una analisi à la Lacan all'architettura, parlando di "politica della verticalità", di un sistema che mira a ricordare costantemente alla popolazione oppressa il suo stato di soggiogamento e subalternità.

A tutto questo si aggiungono gli oltre 600 checkpoint, molti dei quali "volanti" o estemporanei, che contribuiscono a creare uno stato di confusione e incertezza nella vita quotidiana dei palestinesi, i quali possono muoversi sulla base di permessi basati su regole demografiche e burocratiche.

L'antropologo e attivista israelo-ameicano Jeff Halper, fondatore dell'Israeli Committee Against House Demolitions, definisce questo caos strutturato, questo sistema di elementi fisici, architettonici, burocratici e legislativi con il termine omnicomprensivo "matrix of control" (griglia di controllo). Secondo Halper questa griglia ha due obiettivi principali: rompere la contiguità territoriale dei territori palestinesi e paralizzare il più

7 Il dato specifico è stato rilevato dalla ricerca dell'Applied Research Institute of Jerusalem intitolata Israeli Settlement activities swallow more land from Nahalin Village, pubblicata nel 2002. Altre fonti utili per monitorare e verificare la continua espansione degli insediamenti israeliani in Cisgiordania sono i siti delle organizzazioni non governative B'tselem (nella sezione Land expropriation and settlements sul sito <www.btselem.org/topic/settlements) e Alternative Information Center, www.alternativenews.org $>$.

8 Il testo più emblematico di Weizman sull'argomento è Hollow land. Israel's architecture of occupation. 
possibile la vita della popolazione. Io definisco la "matrix of control" una relazione di potere, dal momento che è messa in atto da un Governo nei confronti di una popolazione che, secondo la Quarta Convenzione di Ginevra, dovrebbe essere protetta, proprio a causa del fatto di essere occupata. La relazione di potere così articolata si esprime attraverso un intervento fisico sul paesaggio e sui paesaggi.

\section{L'industria dell'illusione}

A questa sovrapposizione di significati, paesaggi, e segni occorre aggiungere un ulteriore e, in parte, già citato livello: l'industria del turismo. Il termine "industria dell'illusione" applicato a questo settore l'ho carpito e fatto mio durante una conversazione con Adnan Ramadan, educatore e sociologo palestinese, fondatore della rete di organizzazioni OPGAI (Occupied and Golan Heights Advocacy Initiative). Sebbene il suo lavoro non sia a stretto contatto con gli internazionali che si muovono nell'area, il suo sguardo curioso e il gran numero di conferenze che ha tenuto in Europa lo rende un informatore interessante sulla percezione che i non-israeliani e i non-palestinesi hanno della terra, del territorio, della storia.

Per dare un inquadramento quantitativo al fenomeno turistico in Israele e in Palestina si può fare riferimento ad alcuni dati diffusi dal Ministero del Turismo israeliano nel 2010: nei primi nove mesi del 2010 sono entrate nel paese 2,5 milioni di persone. Di questi il 41 per cento attraverso viaggi organizzati da tour operator. Il dato può essere confrontato con quello fornito da Rami Kassis, direttore esecutivo dell'organizzazione palestinese Alternative Tourism Group, che in un'intervista da me effettuata nel 2011, ha dichiarato: "Unendo i dati del Ministero del Turismo palestinese e l'istituto di statistica possiamo dire che 3.000.00o di persone hanno visitato Israele. Di queste 1.800.000 entrano nelle aree palestinesi”. E aggiunge: "Non essendoci frontiere controllate dall'Autorità Nazionale Palestinese il modo per calcolare il numero di visitatori che arrivano in Palestina è quello di contare gli accessi alla Chiesa della Natività e le persone che transitano da Gerico".

Il confronto tra le cifre genera il primo livello di illusione. A questo si aggiunge l'illusione generata dal percorso costruito dalle guide cui i viaggiatori si affidano. Il 41 per cento di viaggi organizzati cui fa riferimento il Ministero del Turismo israeliano ha interesse a promuovere un tipo di esperienza che consolidi l'immagine di Israele come "democrazia del Medio Oriente" e come paese circondato da nemici agguerriti. Il contributo dei turisti alla narrazione più consolidata è sempre stato considerato strategico dallo Stato di Israele. Una dichiarazione del 1973 di Moshe Kol, già Ministro del Turismo, è emblematica delle strategie che ora vengono affidate, con congrui investimenti, alle agenzie di comunicazione internazionali:

I milioni di turisti che hanno visitato Israele sin dalla sua nascita hanno contributo a una migliore comprensione della nostra situazione negli altri paesi. I flussi di denaro che hanno lasciato nel paese non sono meno importanti 
dell'esperienza che si portano via. Quando tornano a casa, nelle loro comunità di appartenenza, tra parenti e amici, diventano ambasciatori di Israele, spargendo ovunque il nostro messaggio. Questo costituisce il nostro passato, e costituirà il nostro futuro. E contribuirà a uno sviluppo considerevole della simpatia dell'opinione pubblica nei nostro confronti. È più vitale per Israele di ogni contributo economico. Inoltre i giovani turisti che vengono a visitarci non solo avranno un'influenza immediata sull'opinione pubblica, ma diventeranno la classe dirigente dei loro paesi. Per questo noi diamo la massima importanza alle loro visite e alla prima impressione. 9

A conferma del perdurare di questo atteggiamento strategico si può citare il documento pubblicato dal Ministero del Turismo e, in contemporanea e non casualmente, dal Ministero degli Affari Esteri israeliani nel novembre del 2010: Tourism investment in Israel. Where unique heritage assets meet a solid business environment. Il pamphlet era stato creato per attirare gli investimenti stranieri, soprattutto per la costruzione di nuove strutture ricettive. Strutture necessarie ad accogliere l'ambiziosa cifra di 5 milioni di turisti entro il 2015. Quattro le linee guida del progetto:

1. sviluppare e promuovere le maggiori attrazioni;

2. consolidare l'immagine di Israele come meta turistica attraverso campagne e operazioni di marketing;

3. fornire assistenza agli imprenditori interessati a costruire alberghi e attrazioni;

4. agevolare l'accesso alla "terra di stato", 10 consentendone l'utilizzo anche senza bandi.

Un esempio che concretizza il contributo della comunità internazionale all'industria dell'illusione è lo strumento che definisce il luogo nella mente e nell'esperienza del viaggiatore: la guida turistica. Lonely Planet è una delle case editrici più famose in questo settore del mercato editoriale. Sebbene non sia possibile calcolare l'esatta quantità di guide dedicate a Israele $e i$ territori palestinesi vendute complessivamente, non è difficile pensare che si tratti di una delle risorse più utilizzate. La visione parziale offerta dalla guida è già presente nel titolo stesso del volume, Israele e $i$ territori palestinesi. ${ }^{11}$ Gli autori stessi nelle righe introduttive della guida spiegano come sia possibile identificare le diverse posizioni politiche in merito all'area sin dal modo di chiamare le diverse parti del territorio. Sebbene gli autori non dichiarino una precisa posizione, il fatto che nel titolo si parli di "territori

9 Il testo è inserito nel saggio di Klein The second million. Israel tourist industry: past, present and future, Amir Publishing.

1o L'accesso alla terra di stato in un contesto come quello israelo- palestinese non si configura solo come una agevolazione, ma come un metodo di conquista spazioterritoriale, con precisi obiettivi politici. Per approfondire si può fare riferimento a numerose ricerche già citate in questo lavoro, ma in particolare allo studio dell'organizzazione israeliana B'tselem <www.btselem.org/topic/settlements $>$.

11 Edizione italiana del 2008 della guida Lonely Planet dedicata a Israele e i Territori Palestinesi 
palestinesi" senza utilizzare l'aggettivo "occupati” diventa una precisa dichiarazione politica. In questo modo si decide di non problematizzare la contesa, interna e internazionale, sulla definizione di occupazione militare della Cisgiordania della Striscia di Gaza.

Il secondo criterio che identifica la guida Lonely Planet come sbilanciata verso la narrazione israeliana della storia e della geografia si individua nella descrizione di Gerusalemme.

Nel 1967 la "Guerra dei Sei Giorni" ha portato alla riunificazione di Gerusalemme. ${ }^{12}$

Sebbene Gerusalemme sia la capitale indiscussa dello Stato di Israele, le stranezze della diplomazia internazionale hanno indotto molti stati a insediare le loro ambasciate a Tel Aviv. ${ }^{13}$

Qualunque lettore informato sulla controversia storico - geografica nota come "dibattito sullo status di Gerusalemme" resterà stupito dalla semplificazione con cui il turista viene avvicinato alla città. La questione è rilevante a tal punto da essere una delle chiavi del processo di pace e del suo stesso stallo, insieme alla questione del rientro dei profughi ${ }^{14}$ e alla gestione degli insediamenti. Per comprendere la portata geopolitica della dichiarazione o meno di Gerusalemme come capitale si può fare riferimento a numerose risoluzioni del Consiglio di Sicurezza delle Nazioni Unite, la più famosa la numero 478 del 1980.15

\section{Smarrirsi per sfuggire al controllo}

Sebbene il paesaggio sia complesso, storicamente relativo e contestato, lo si può comunque considerare un testo utilizzando Roland Barthes come grimaldello interpretativo. Un testo può essere infatti letto secondo differenti interpretazioni. Ł̇ proprio la decodifica l'azione che Halper suggerisce ai palestinesi come mezzo di resistenza: secondo l'antropologo infatti solo la consapevolezza di essere chiusi in bantustan ${ }^{16}$ può aiutare e determinare la lotta per l'autodeterminazione.

In questo processo di decodifica il ruolo del corpo e del movimento diventa fondamentale, soprattutto per il "turista", soggetto a molte meno

12 Pagina 85, ibidem.

13 Pagina 429, ibidem.

14 Della questione dei profughi si fa cenno anche nel quarto paragrafo, in riferimento alla definizione, alla percezione e all'accesso a Israele da parte dei figli delle persone allontanate dall'area nel 1948 e nel 1967 (v. nota 21).

15 Oltre alla risoluzione del Consiglio di Sicurezza delle Nazioni Unite, accessibile nella sua versione ufficiale consultando la sezione del sito delle Nazioni Unite intitolata The Question of Palestine <unispal.un.org/UNISPAL.NSF/o/DDE590 C6FF232007852560DFo065FDDB > [accesso effettuato il 10 giugno 2014] si può fare riferimento a un'ampia letteratura. In bibliografia è citato Vittime. Storia del conflitto arabo-sionista. 1881- 2001 dello storico ebreo israeliano Benny Morris.

16 Jeff Halper nell'articolo Dismantling the matix of control del 1999 paragona esplicitamente le aree A dei Territori Palestinesi ai bantustan sudafricani, cioè le porzioni di territorio assegnate alle etnie nere durante l'apartheid. 
regole rispetto a quelle di cui sono vittime per certi versi gli israeliani e quasi completamente i palestinesi. Il corpo può trascendere la narrativa di massa $\mathrm{e}$ camminare sul ponte immaginario che c'è tra la concezione tipicamente occidentale dello stato di Israele e il ritratto ingannevole perpetrato dall'orientalismo ${ }^{17}$ nel raccontare la popolazione palestinese. Infatti il turista ha un passaporto che gli permette di muoversi tra spazi e luoghi, dall'una all'altra delle sei dimensioni di cui parlava Benvenisti. Attraverso i suoi movimenti il turista può assaporare la libertà che vive il suo corpo, sia nella vita quotidiana, sia quando messa a confronto con la vita quotidiana della popolazione locale. Il popolo palestinese, come collettività e come singoli individui, non può muoversi liberamente da Betlemme a Gerusalemme, quando cammina per le strade della città di Al Khalil - Hebron ${ }^{18}$ si trova davanti reti e blocchi, deve attraversare cinque checkpoint per entrare nella Moschea di Ibrahim, a cui corrisponde anche una sinagoga a uso esclusivo dei coloni. Un popolo che, su quello che è il confine immaginario tra Israele $\mathrm{e}$ Cisgiordania, noto come Linea Verde era abituato a vedere campi di ulivi e ora vede lastre di cemento alte otto metri. Proprio l'area oltre queste lastre e questo confine concreto e immaginato diventerà rilevante nella parte finale di questo saggio racconto

Per vivere questo tipo di esperienza-ponte il "turista" ha due possibilità. Una organizzata e l'altra apparentemente casuale, l'una ascrivibile all'industria del turismo, l'altra a un atteggiamento "situazionista".

Un settore particolare dell'industria del turismo è il cosiddetto turismo alternativo. In un manuale messo a punto dalla già menzionata organizzazione israelo- palestinese Alternative Information Center vengono descritti i diversi tipi di tour, definiti come "alternativi, critici, esperienziali $e$ impegnati". Proprio l'ultima categoria è quella con una descrizione particolarmente adatta a quanto si sta cercando di dimostrare:

Turismo impegnato significa un'esperienza che abbatte i confini tra popolazione locale e visitatori, permettendo al visitatore di partecipare alle attività di costruzione della giustizia. Tutti i turisti hanno un impatto sulla popolazione locale, sia attraverso i semplici acquisti che fanno che attraverso i racconti che riportano a casa. Nel "turismo impegnato" i turisti non abbandonano i loro interessi, impegni e infrastrutture ma li integrano con l'esperienza di apprendimento, che fa parte del piacere e del relax. Accanto alle tre "S" del viaggio (see, sun and scenery -

17 In questo saggio non viene argomentata e descritta la critica all'orientalismo fatta da Edward Said nel suo libro del 1979 Orientalismo. Si può sintetizzare l'analisi di Said nella frase: “L’Oriente, gli Arabi e l'Islam, separati o insieme, sono stati congelati in uno stato fisso, come un oggetto ghiacciato e posto una volta per tutte sotto le lenti dello sguardo occidentale".

18 La città di Hebron, antico cuore economico della Cisgiordania ha progressivamente acquisito i contorni di una città fantasma. I due nomi corrispondono al toponimo della città a seconda che venga considerata ebraica o palestinese. Si tratta dell'unico caso (insieme alla città vecchia di Gerusalemme) in cui i coloni ebrei vivono in mezzo alla popolazione palestinese. Si può dire che alle sei dimensioni identificate da Benvenisti se ne aggiungono in questo caso altre due: una dimensione "nominale", il doppio toponimo, e una dimensione "religiosa", la Sinagoga e la Mosche che occupano lo stesso luogo fisico e spaziale. 
traduzione: vedere, sole e scenario, le tre caratteristiche che accomunano ogni viaggio) i turisti cercano di imparare qualcosa della cultura locale e delle questioni sensibili.

Nel 1947 la Società delle Nazioni definì come turismo "genti che viaggiano per periodi di oltre 24 ore". Questa definizione ci accompagna verso la conclusione di questo saggio, l'approccio situazionista allo smarrimento che diventa un rischio inevitabile nel complesso di interpretazioni e griglie di controllo e potere descritte fino a questo momento.

L'aneddoto che segue proviene dal diario di campo che ho tenuto durante la mia ricerca etnografica sul viaggio in Israele e Palestina nell'estate del 2011.

Un sabato di agosto mi trovavo con I. e A. in macchina per una gita di un giorno a Jenin, una città di 35.000 abitanti nel nord della Cisgiordania.

I. è italiana, ha 27 anni e lavora in una ong di Beit Sahour, un paese di 10.000 abitanti vicino a Betlemme. A. è palestinese, ha 29 anni e lavora occasionalmente presso la stessa ong. Abbiamo affittato una macchina (con la targa verde ${ }^{19}$ ) a Beit Sahour. Lungo la strada fino a Ramallah non abbiamo problemi a orientarci. Conosciamo la strada e i segnali stradali sono piuttosto chiari. Per quello che sappiamo dobbiamo arrivare nei pressi della città di Nablus, superare il checkpoint noto come Huwara, unica via di accesso alla città e poi proseguire fino a Jenin. Il problema è che da Ramallah in poi non troviamo nessun segnale stradale. Riusciamo ad arrivare a Nablus, ma ci ritroviamo su una strada di quelle che vengono definite by pass road, si tratta dei collegamenti tra $\mathrm{i}$ diversi insediamenti israeliani in Cisgiordania. I segnali stradali indicano che stiamo superando la la grande colonia di Ariel, con i suoi $\mathbf{1 6 . 0 0 0}$ abitanti e la sua università. Potremmo trovare con facilità la Riserva Naturale israeliana, ben indicata. Lungo la strada le indicazioni per arrivare qui sono chiare, non si trovano invece né i nomi dei villaggi arabi che pur costellano la zona, né le indicazioni per arrivare a Jenin. Il risultato è che dopo diversi kilometri da Huwara, nostro ultimo riferimento, siamo completamente smarriti.

A pochi metri da noi appare un checkpoint in muratura, si tratta della Green Line. Non abbiamo nessuna possibilità di superarlo. Per due motivi: la nostra targa, verde, non ci dà l'autorizzazione. $\mathrm{E}$ A. ha una carta di identità anch'essa verde: in quanto residente dei Territori Palestinesi non ha l'autorizzazione per passare. ${ }^{20}$

Il checkpoint sembra più un casello autostradale. Il personale di sicurezza, munito di fucili mitragliatori, ci chiede di spiegare la nostra presenza sul posto. Inizialmente $\mathrm{i}$ fucili ci vengono puntati addosso. Una volta che le guardie

19 Nei Territori Palestinesi Occupati le macchine dei palestinesi sono contraddistinte da una targa verde, diversa da quella gialla che identifica le macchine appartenenti a cittadini israeliani e residenti di Gerusalemme. A seconda del tipo di targhe le macchine hanno diverse possibilità di accesso alle strade a percorrenza veloce.

20 Nel contesto descritto anche i documenti di identità giocano un ruolo pratico e simbolico, così come le targhe: i residenti dei Territori Palestinesi hanno un documento verde, che non permette loro di oltrepassare la Green Line salvo dietro presentazione di un permesso rilasciato con un certo anticipo. Significa che non possono recarsi né a Gerusalemme né all'interno di Israele. 
comprendono che abbiamo semplicemente sbagliato strada diventano più gentili, ci invitano a scendere dalla macchina e controllano i nostri documenti. La macchina viene perquisita meticolosamente. Noto che mentre I. e io siamo piuttosto tese, A. sembra quasi sorridente. Ci vengono restituiti i documenti e per fare inversione a $U$ siamo autorizzati a superare il checkpoint e - di fatto - a superare la Linea Verde. Proprio mentre ci troviamo al di là della linea di confine e stiamo tirando un sospiro di sollievo vedo A. comporre frettolosamente un numero. Parla in arabo ma capisco che sta parlando con Y., un suo amico di Beit Sahour. Urla con entusiasmo: "Indovina? Sono in 48 ".

I palestinesi tendono a non chiamare "Israele" il territorio posto oltre la Linea Verde. Soprattutto gli attivisti definiscono l'area "48". 1948 è l'anno della fondazione dello Stato di Israele e della prima guerra tra i paesi arabi, i palestinesi e Israele. L'anno che per Israele segna l'indipendenza e i palestinesi chiamano nakba (traduzione dall'arabo catastrofe). Il turista ha la possibilità di vivere e praticare col corpo i due lati del muro. Questo significa che vede gli otto metri di cemento quando si trova dal lato "palestinese" e gli alberi e il terrapieno che si percepisce dal lato "israeliano" quando si percorre la strada che costeggia il muro.

Per A. essere in " 48 " è una conquista. Significa aver valicato un confine per lui inaccessibile e significa riappropriarsi di un pezzo della sua identità. Infatti durante le guerre tra il 1948 e il 1967 centinaia di villaggi palestinesi che si trovavano dove ora si trovano le città israeliane sono stati distrutti. Le famiglie palestinesi che abitavano lì sono diventate profughe. $\mathrm{E}$ da allora ambiscono a rientrare nelle loro terre e nelle loro case. Una delle immagini più potenti della storia contemporanea palestinese è quella della chiave. Quasi tutte le famiglie nei campi profughi conservano la chiave delle case abbandonate durante la nakba. Sebbene esista una risoluzione dell'Assemblea Generale delle Nazioni Unite che sancisce il "diritto al ritorno" le politiche sui permessi e le infrastrutture architettoniche impediscono questo rientro ${ }^{21}$.

L'episodio dimostra come l'industria dell'illusione e del potere descritta fino a questo punto possa in qualche modo essere superata. La tecnica è quella che i situazionisti chiamavano derivé. Con questo termine si definiva un "comportamento costruttivo e giocoso", che mira a costruire una "consapevolezza psicogeografica dello spazio". La tecnica, così come descritta da Guy Debord, si basava su lunghe camminate nello spazio, tentando di evitare percorsi predeterminati, sia reali che simbolici. Questo approccio ben si adatta, con le debite proporzioni e contestualizzazioni, a quanto vissuto dai passeggeri della macchina ribelle. È lo stesso invito che l'avvocato e scrittore palestinese Raja Shehadeh fa nella sua raccolta di racconti Palestinian Walks. Notes on a vanishing landscape del 2008. L'avvocato racconta la storia della Palestina del passato, del presente e del futuro attraverso sette sarhat. In arabo il termine descrive "un uomo che

21 Risoluzione 194 <http://unispal.un.org/UNISPAL.NSF/o/C758572B78D1CDoo 85256BCFoo77E51A $>$ [accesso effettuato il 28 maggio 2013]. 
cammina senza scopo, senza restrizioni di spazio e di tempo. Un uomo che va dove lo porta lo spirito, dove l'anima si nutre e ringiovanisce". Nell'introduzione alla raccolta l'autore invita il lettore a prendere parte a questo processo di ricostruzione di un paesaggio evanescente.

L'esperienza sulla Green Line vissuta da I., A. e me, con Y. in collegamento telefonico è la riposta all'invito di Shehadeh al suo lettore. L'invito a un movimento che concretizzi l'“arte della memoria” di Gian Battista Vico citata nell'antropologia del paesaggio di Hirsch: un'esperienza condivisa che aiuti a costruire conoscenza, una conoscenza che fugge da costruzioni predefinite. Il turista, il viaggiatore e il palestinese si prendono per mano per sfuggire al caos strutturato e per decodificarlo oltre i segnali stradali, le barriere e le costruzioni e gli investimenti e le campagne promozionali dell'industria del turismo. 


\section{Bibliografia}

AA.VV.

1999 Alternative tour guide training, Jerusalem, The Alternative Information Center.

Bender, Barbara

2001 Introduction in B. Bender, M. Winer Contested landscapes. Movement, exile and place, Oxford, Berg.

Benvenisti, Meron

2000 Sacred Landscape. The buried history of the Holy Land since 1948, Berkeley, University of California Press.

Boussac, Julien

2009 “Eastern Palestine Archipelago”, in Atlas Du Monde Diplomatique.

Debord, Guy

1956 "Theory of the Derivè", in Les Lèvres Nues \#9 (November 1956) (ristampato in Internationale Situationniste \#2 (December 1958) <www.cddc.vt.edu/sionline///si/theory.html > [accesso effettuato il 10 giungo 2014].

Fiume, Virginia

2011 From Holy Land to Palestine: the role of "international committed people in the Plaestinian struggle, London, Soas $-<$ www.virginiafiume.com $>$.

Gelvin, James

2005 The Israel- Palestine conflict: one hundread years of war, Cambridge, Cambridge University Press.

Halper, Jeff

1999 "Dismantling the Matrix of Control", in News from within, vol. XV, n. 9, October 1999.

Hirsch, Emil

1999 "Introduction. Landscape between place and space", in E. Hirsch, M. O'Hanlon (eds.) The Anthropology of landscape. Perspective on place and space, Oxford, Clarendon Press.

Klein, C.H.

1973 The second million. Israel tourist industry: past- present- future, Israele, Amir.

Kohn, Michael

2008 Israele e i Territori Palestinesi, Torino, Lonely Planet - StampaTre.

Ministry of tourism of Israel

14 | www.ocula.it | Giugno 2014 
2011 Tourism investment in Israel. Where unique heritage assets meet a solid business envaironment [online] http://<www.goisrael.com/other/flashbooks/all/tourisminvestmentinisrael/f iles/assets/basic-html/page1.html > [accesso effettuato il 10 giungo 2014].

Morris, Benny

2003 Vittime. Storia del conflitto arabo-sionista. 1881- 2001, Milano, Rizzoli.

Selwyn, Tom

2001 "Landscapes of separation: reflection on the symbolism of by-pass roads in Palestine", in B. Bender, M. Winer (eds.) Contested landscapes. Movement, exile and place, Oxford, Berg.

Shehadeh, Raja

2008 "Introduction", and "And how did you get over it?", in Palestinian walks. Notes on a vanishing landscape, London, Profile Books Ltd.

Stein, Rebecca

2008 "Of cafés and terror", in Itineraries in conflict. Israelis, Palestinians, and the political lives of tourism, USA: Duke University Press.

Thomas, Julian

2001 "Intersected landscapes", in B. Bender, M. Winer (eds.) Contested landscapes. Movement, exile and place, Oxford, Berg.

Weizman, Eyal

2007 Hollow Land. Israel's architecture of Occupation, London, Verso.

Zureik, Elia

2011 "Colonialism, surveillance, and population control: Israel/ Palestine", in E. Zureik, D. Lyon, Y. Abu-Laban (eds) Surveillance and control in Israel/ Palestine. Population, territory and power, London. Routledge.

Zonszein, M.

2014 Walled off: 12 years of Israel's separation barrier in AlJazeer America, $<$ america.aljazeera.com/articles/2014/3/12/visual-activismactivestillsphotographsthebarrierwall.html> [accesso effettuato il 10 giugno 2014] 